\title{
RECURSIVE DENSITY TYPES. I
}

\author{
BY \\ H. GONSHOR AND G. RICE
}

1. Introduction. The theory of recursive density types was introduced by Medvedev [1] and studied by Pavlova [2], [3]. Rice [4] made use of the concept in order to characterize $B^{*}$, the class of recursively bounded orders. In this paper we shall develop an algebra of recursive density types (called densities for short) analogous to Dekker's theory of recursive equivalence types. In order to keep this work self-contained, theorems probably proved by Pavlova [3] in her paper which was unavailable to us will be proved here. It will be seen that interesting phenomena occur here which do not occur in more traditional cardinality theories.

As far as possible, Dekker's notation for numbers, sets, and classes will be used. In addition the following notations will be adopted. If $\alpha$ is an infinite set, then $\alpha(n)$ is the function enumerating $\alpha$ in order of size. Sometimes $\alpha$ will be used to represent the function providing there is no danger of confusion. Also $n(\alpha, x)$ will denote the number of elements in $\alpha$ not greater than $x$.

2. Definition and elementary properties of densities. Definition

$$
\beta \geqq \alpha \leftrightarrow(\exists \text { recursive } h)(\forall x)\{n[\beta, h(x)] \geqq n(\alpha, x)\} .
$$

Several consequences follow immediately from the definition.

(1) $h$ may be taken to be strictly increasing.

(2) It is sufficient to require the inequality only for all $x \geqq x_{0}$ where $x_{0}$ is fixed.

(3) If $\alpha$ and $\beta$ have finite cardinality, then $\beta \geqq \alpha \leftrightarrow \operatorname{card} \beta \geqq \operatorname{card} \alpha$. If $\alpha$ has finite cardinality and $\beta$ does not, then $\beta \geqq \alpha$ but $\neg(\alpha \geqq \beta)$.

(4) For infinite $\alpha$ and $\beta$ the definition can be expressed equivalently in function language as

$$
\beta \geqq \alpha \leftrightarrow(\exists \text { recursive } n)(\forall n)[\beta(n) \leqq h \alpha(n)] .
$$

Remarks analogous to (1) and (2) hold here also; in fact, it is advisable to use (1) in proving the equivalence of (4) and the original definition.

(5) $\alpha \subset \beta \rightarrow \beta \geqq \alpha . \quad(\forall n)[\beta(n) \leqq \alpha(n)] \rightarrow \beta \geqq \alpha$.

Since by (3) the behavior of finite sets is as trivially expected we shall be concerned primarily with infinite sets and thus (4) will be applicable.

$\geqq$ is a reflexive and transitive relation, hence by partially ordered set theory, if $\alpha \sim \beta$ is defined as " $\alpha \geqq \beta$ and $\beta \geqq \alpha$ ", then $\sim$ is an equivalence relation and the

Received by the editors June 20, 1967 and, in revised form, May 17, 1968. 
equivalence classes which we shall call densities form a partially ordered set $\Delta$ in the induced ordering. As usual, $\bar{\alpha}$ denotes the class containing $\alpha$. It is clear from remark (5) that $\bar{\varepsilon}$ is the maximum element of $\Delta$ where $\varepsilon$ is the set of all nonnegative integers. By (3), the class generated by a finite set can be identified with the cardinality of that set, and then $\geqq$ agrees with its usual meaning for numbers. Such classes will be called finite and all others infinite. Again from remark (3), every finite class is less than every infinite class.

We shall now start gradually getting less trivial. Note first that a rephrasing of one of the definitions for hyperimmune sets is that $\bar{\varepsilon}$ is exactly the class of nonhyperimmune sets. In case this might suggest that classes are too big to be of interest, we also have the result that if $\alpha$ is hyperimmune and $n \in \alpha$ then

$$
\bar{\alpha} \neq \overline{\alpha-(n)}
$$

i.e. deletion of one element is enough to decrease the type. This has been shown several times in the literature (pre-Pavlova) and is easy to see. As a hint to the reader, the basic idea of the proof is that the family of recursive functions is closed under the induction schema.

3. Comparison with other relations in the literature. Note first that every degree of unsolvability has a nonhyperimmune representative. Thus it is possible that $\bar{\alpha}=\bar{\beta}$ with $\alpha$ and $\beta$ being of different degrees and that $\bar{\alpha} \neq \bar{\beta}$ with $\alpha$ and $\beta$ being of the same degree. Furthermore, the existence of immune sets which are not hyperimmune shows that it is possible that $\bar{\alpha}=\bar{\beta}$ but rec. $(\alpha) \neq$ rec. $(\beta)$ in the sense of Dekker. The rest of this section will be used to show that it is possible that $\bar{\alpha} \neq \bar{\beta}$ but rec. $(\alpha)=\operatorname{rec} .(\beta)$.

More specifically we shall prove the following:

THEOREM I. There exists a hyperimmune set which is recursively equivalent to a nonhyperimmune set. (Remark: It is interesting to note, in contrast, that the property of being immune as well as that of being hyper-hyperimmune is preserved under recursive equivalence.)

Proof. Note first that every partial recursive function is bounded by a partial recursive 1-1 function. In fact, if the sequence of pairs $\left(x_{n}, y_{n}\right)$ represents a recursive generation of the given partial recursive function, then $\left[x_{n},\left(\max _{1 \varliminf_{n}} y_{i}\right)+n\right]$ represents a partial recursive 1-1 function bounding the given function. This remark will be used at the end of the proof. We now study Gödel numbers and a special recursive functional. We shall use $q_{n}$ for the standard enumeration of the partial recursive function.

Let $[T f](n)=\left[\max _{m \leqq n} f(m)\right]+n+1$. Then $[T f](n)>f(n)$ for all $n$ and $T f$ is strictly increasing. Since $T$ is a recursive functional, there exists a recursive function $h$ such that $T\left(q_{n}\right)=q_{h(n)}$ for all recursive $q_{n}$. $h$ can be chosen so that $h(n)>n$ and $h(n+1)>h(n)$ for all $n$. Let $a$ be a Gödel number of the function $f$ where $f(n)=n$ 
for all $n$. Define $r$ by $r(0)=a$ and $r(n+1)=h r(n)$. Then $r$ is strictly increasing since $h(n)>n$.

Now choose $x_{n} \leqq r(n)$ so that $q_{x_{n}}$ is strictly increasing recursive, and for all $y \leqq r(n)$ such that $q_{y}$ is strictly increasing recursive $q_{x_{n}}\left(x_{n}\right) \geqq q_{y}(y)$. Note that since $q_{r}(0)=q_{a}, x_{n}$ is defined for all $n$.

Lemma 1. $r(n)<x_{N+1}$. Hence $x_{n}<x_{n+1}$.

Proof. Suppose $y \leqq r(n)$ is an index of a recursive function. Then $h(y) \leqq h r(n)$ $=r(n+1)$. Hence $h(y)$ is a candidate for the choice of $x_{n+1}$. Now $q_{h(y)}[h(y)]$ $>q_{h(y)}[y]>q_{y}[y]$ since $q_{y}(y)$ is strictly increasing, $h(y)>y$, and $q_{h(y)}(n)>q_{y}(n)$ for all $n$. Hence $y \neq x_{n+1}$. This proves the lemma.

LEMMA 2. $q_{x_{n}}\left(x_{n}\right)$ is strictly increasing.

Proof. From the proof of the previous lemma, $q_{h\left(x_{n}\right)}\left[h\left(x_{n}\right)\right]>q_{x_{n}}\left(x_{n}\right)$. Hence a fortiori $q_{x_{n+1}}\left(x_{n+1}\right)>q_{x_{n}}\left(x_{n}\right)$.

Now comes the critical point of the argument. Since $x_{n} \leqq r(n), x_{n}$ is recursively bounded.

LEMMA 3. $q_{x_{n}}\left(x_{n}\right)+1$ is not a recursively bounded function of $n$.

Proof. Let $y$ be an index of a strictly increasing recursive function. By Lemma 2 it suffices to find an $n$ such that $q_{x_{n}}\left(x_{n}\right)+1>q_{y}(n)$. Note that there is no loss in generality in assuming that $y>r(0)$ since every function has infinitely many indices. Then $(\exists n)(r(n-1)<y \leqq r(n))$.

By choice of $x_{n}, q_{x_{n}}\left(x_{n}\right) \geqq q_{y}(y)$. Since $r$ is strictly increasing, $y \geqq n$. Since $q_{y}$ is strictly increasing, $q_{y}(y) \geqq q_{y}(n)$. Hence $q_{x_{n}}\left(x_{n}\right) \geqq q_{y}(n)$. This proves that $q_{x_{n}}\left(x_{n}\right)$ $+1>q_{y}(n)$. Q.E.D.

Thus we found a partial recursive function $q_{x}(x)+1$ which maps a recursively bounded set onto a set which is not recursively bounded. We now make our promised use of a remark made earlier and conclude that $q_{x}(x)+1$ is bounded by a partial recursive 1-1 function $P(x) . P\left(x_{n}\right)$ also is not a recursively bounded function of $n$. This is obvious but not absolutely obvious since $P\left(x_{n}\right)$ is not necessarily increasing as a function of $n$.

However, the $n$th term of the set $P\left\{x_{n}\right\}$ arranged in order of size must be at least $q_{x_{n}}\left(x_{n}\right)+1$. (Throughout this discussion it is important not to confuse $f(x)$ as a function of $x$ with $f\left(x_{n}\right)$ as a function of $n$.)

Thus we have finally shown that $P$ sets up a recursive equivalence between the nonhyperimmune set $\left\{x_{n}\right\}$ and the hyperimmune set $P\left\{x_{n}\right\}$. This proves the theorem.

Note. Incidentally, by the remarks at the end of the statement of the theorem we have constructed, as a by-product, an immune set $\left\{x_{n}\right\}$ which is not hyperimmune and a hyperimmune set $P\left\{x_{n}\right\}$ which is not hyper-hyperimmune. An example of the former is due to Post and of the latter to Yates. 


\section{Lattice properties.}

THEOREM 2. The densities form a lattice with

$$
\bar{\alpha} \cup \bar{\beta}=\overline{\min [\alpha(n), \beta(n)]}
$$

and

$$
\bar{\alpha} \cap \bar{\beta}=\overline{\max [\alpha(n), \beta(n)]} .
$$

(Note that the function form is used in the definition. To those familiar with functional analysis, the min and max might appear to be reversed, but it must be remembered that the larger the function, the smaller the density. Also the rule does not cover the finite elements but their behavior is trivial anyway.)

Proof. It suffices to work with the representatives $\alpha, \beta$, and $\min (\alpha, \beta)$. Clearly $\min (\alpha, \beta) \geqq \alpha$ and $\min (\alpha, \beta) \geqq \beta$. (h can even be taken to be the identity function.) Let $\gamma \geqq \alpha$ and $\gamma \geqq \beta$. Then $\exists$ recursive $h_{1}, h_{2}$ such that $\gamma(n) \leqq h_{1} \alpha(n)$ and $\gamma(n) \leqq h_{2} \alpha(n)$. Let $h=\max \left(h_{1}, h_{2}\right)$. Then $h$ is recursive. Also $\gamma(n) \leqq h \alpha(n)$ and $\gamma(n) \leqq h \beta(n)$. Hence $\gamma(n) \leqq h\{\min [\alpha(n), \beta(n)]\}$. Thus $\gamma \geqq \min (\alpha, \beta)$.

Again, $\alpha \geqq \max (\alpha, \beta)$ and $\beta \geqq \max (\alpha, \beta)$. Let $\alpha \geqq \gamma$ and $\beta \geqq \gamma$. Then $\exists$ recursive $h_{1}, h_{2}$ such that $\alpha(n) \leqq h_{1} \gamma(n)$ and $\beta(n) \leqq h_{2} \gamma(n)$. Hence

$$
\max [\alpha(n), \beta(n)] \leqq \max \left[h_{1} \gamma(n), h_{2} \gamma(n)\right]=h \gamma(n)
$$

where $h=\max \left(h_{1}, h_{2}\right)$. Hence, $\max (\alpha, \beta) \geqq \gamma$.

COROLlaRY. The lattice is distributive with $\bar{\varepsilon}$ as $a 1$ and $\bar{\Lambda}$ as $a 0$.

Note 1. The symbol " 1 " will be reserved for the class of all unit sets. Fortunately, in the case of " 0 ", both the lattice and cardinality interpretation agree.

Note 2. In the theorem, any recursive function $h$ of two variables such that $h(m, n) \geqq \max (m, n)$ can be used instead of max to determine the intersection.

Note 3. The lattice is certainly not a Boolean algebra.

Corollary. If $B \geqq A$ and $\bar{\alpha}=A$ then $\exists \beta(\bar{\beta}=B)$ and $\forall n[\beta(n) \leqq \alpha(n)]$. Similarly if $B \geqq A$ and $\bar{\beta}=B$, then $(\exists \alpha)(\bar{\alpha}=A)$ and $\forall n[\beta(n) \leqq \alpha(n)]$.

These are trivial but convenient results to have. For example in part 1 if $\beta$ is any representative of $B$ then $\min [\beta(n), \alpha(n)]$ is a representative of $B \cup A=B$ by the theoren. Hence it can serve as the required $\beta$. A similar argument applies to part 2.

We now arrive at an important density theorem.

THEOREM 3. If $A$ and $B$ are infinite, $A<B \rightarrow(\exists H)(A<H<B)$. In fact there are $c$ mutually.incomparable as well as $c$ linearly ordered densities strictly between $A$ and $B$.

REMARK. This result certainly illustrates that in spite of the size of the single class $\bar{\varepsilon}, \Delta$ is quite rich. 
Proof. Choose representatives $\alpha \in A$ and $\beta \in B$ such that $\beta(n) \leqq \alpha(n)$ for all $n$. We assume for convenience that if $B=\bar{\varepsilon}$, then $\beta(n)=2 n$. Furthermore, since $A<B$ it follows that $(\forall$ recursive $f)(\exists n)[\alpha(n)>f \beta(n)]$ and by an earlier remark even that $(\forall$ recursive $f)\left(\forall n_{0}\right)(\exists n)\left[n>n_{0}\right.$ and $\left.\alpha(n)>f \beta(n)\right]$.

Consider the class of all pairs $(f, l)$ where $f$ is a recursive function and $l$ is a number. Since this class is countable it can be arranged in a sequence $\left\{\left(f_{i}, l_{i}\right)\right\}$. We shall construct a sequence $m_{i}$ of numbers as follows:

Let $m_{0}$ satisfy $\alpha(n)>f_{0} \beta(n)$. Now suppose $m_{i}$ has been defined. As a preliminary technical point note that for $m$ sufficiently high $\alpha\left(m_{i}\right)+m \leqq \beta_{i}\left(m_{i}+m\right)$. [Otherwise $\beta$ is recursively bounded. Furthermore, even that case is ruled out by the choice $\beta(n)=2 n$ and by the trivial remark that the function $f(n)=a+n$ for a constant $a$ is not an upper bound to the function $f(n)=2 n$.] Now choose $m_{i+1} \geqq m_{i}+m$ such that $\alpha\left(m_{i+1}\right)>f_{i+1} \beta\left(m_{i+1}\right)$.

Let $c=\left\{A_{i}\right\}$ be an arbitrary sequence of 0 's and 1's. We define a function $\gamma_{c}$ as follows:

$$
\begin{gathered}
\text { if } n<m_{0}, \quad \gamma_{c}(n)=\beta(n) \\
\text { if } m_{i} \leqq n<m_{i+1} \quad \text { and } \quad c_{l_{i}}=1, \quad \gamma_{c}(n)=\beta(n) \\
\text { if } m_{i} \leqq n<m_{i+1} \text { and } c_{l_{i}}=0, \\
\gamma_{c}(n)=\max \left[\alpha\left(m_{i}\right)+\left(n-m_{i}\right), \beta(n)\right] .
\end{gathered}
$$

Note first that $\beta(n) \leqq \gamma_{c}(n) \leqq \alpha(n)$. This follows since $\alpha$ is strictly increasing. Furthermore, $\gamma_{c}(n)$ is strictly increasing. In fact the sole purpose of the technical point mentioned earlier is to ensure this in case $\gamma_{c}\left(m_{i}\right)=\alpha\left(m_{i}\right)$ and $\gamma_{c}\left(m_{i+1}\right)=\beta\left(m_{i+1}\right)$. Intuitively speaking, $\gamma_{c}$ is going up slowly in order to let $\beta$ catch up.

We now show the critical fact that $(\forall i)\left(c_{i} \geqq d_{i}\right) \leftrightarrow \gamma_{c} \geqq \gamma_{d}$ where the right hand inequality is used in the density sense.

If $\forall_{i}\left(c_{i} \geqq d_{i}\right)$, then $d_{i}=1 \rightarrow c_{i}=1$. Hence it follows immediately from the formula that $\forall n\left[\gamma_{c}(n) \leqq \gamma_{d}(n)\right]$. This implies that $\gamma_{c} \geqq \gamma_{d}$ in the density sense.

If $\exists i\left(c_{i}<d_{i}\right)$ then choose $j$ so that $d_{j}=1$ and $c_{j}=0$. Let $g$ be an arbitrary recursive function. Then $(\exists i)\left(f_{i}=g\right.$ and $\left.l_{i}=j\right)$. By the formula $\gamma_{c}\left(m_{i}\right)=\alpha\left(m_{i}\right)$ and $\gamma_{d}\left(m_{i}\right)=\beta\left(m_{i}\right)$. Therefore $\gamma_{c}\left(m_{i}\right)=\alpha(m)>f_{i} \beta\left(m_{i}\right)=g \gamma_{d}\left(m_{i}\right)$. Since $g$ is arbitrary this shows that $\gamma_{c} ¥ \gamma_{d}$ in the density sense.

It now follows that the map $c \rightarrow \bar{\gamma}_{c}$ is an order preserving mapping from the class of sequences of 0 's and 1's with its usual partial order into $\Delta$. Also $A \leqq \bar{\gamma}_{c} \leqq B$. Since order is preserved both ways the map is $1-1$. The problem has now been reduced to one in ordinary set theory. Note, incidentally, that the possibility that $\gamma_{d}=A$ or $B$ for some $d$ is no problem since subtracting at most 2 elements from a set of cardinality $c$ still leaves a set of cardinality $c$.

The class of sequences with the usual partial order is order isomorphic to the class of all subsets of the integers where $\leqq$ is ordinary set inclusion. The proof of the existence of a linearly ordered class of subsets of cardinality $c$ is a well-known 
classical exercise. The proof of the existence of $c$ mutually incomparable subsets is simpler and is a good exercise for a graduate student. This completes the proof of Theorem 3.

It is clear that the mapping $c \rightarrow \bar{\gamma}_{c}$ is not onto the set of all densities $H$ satisfying $A \leqq H \leqq B$. In fact, to show this we need only to reapply the previous theorem to $\bar{\gamma}_{c}$ and $\bar{\gamma}_{d}$ where $\neg(\exists x)(c>x>d)$. Thus it does not follow from pure lattice theory that the map preserves the lattice operations. However, this follows immediately from the definition of $\gamma_{c}$ and from Theorem 2. We have shown that between any two infinite elements in $\Delta$ there is a sublattice isomorphic to the Boolean algebra of subsets of a countable set. Incidentally it follows immediately that if the smaller density is finite and the larger remains infinite all the results are still valid.

5. Addition and multiplication. It is convenient to use sets rather than functions for the definitions of the algebraic operations. Addition will be defined as follows: If $A$ and $B$ are two densities let $\alpha$ and $B$ be representatives of $A$ and $B$ respectively such that $\alpha \cap B=\varnothing$. Then

$$
A+B=(\overline{\alpha \cup B}) .
$$

Note first that as opposed to the theory of R.E.T.'s separability conditions are not required. Secondly, although the results in $\$ 3$ suggest caution, it is still true that disjoint representatives always exist. In fact $\{\alpha(n)\} \sim\{2 \alpha(n)\}\{2 \sim \alpha(n)+1\}$. We now show that the operation is well defined.

LEMmA. If $\alpha \cap \beta=\alpha \cap \gamma=\varnothing$ and $\beta \geqq \gamma$ then $\alpha \cup \beta \geqq \alpha \cup \gamma$.

Proof. Choose a strictly increasing recursive $h$ such that

$$
(\forall x)\{n[\beta, h(x)] \geqq n(\gamma, x)\} .
$$

Since $h(x) \geqq x$ clearly $n[\alpha, h(x)] \geqq n(\alpha, x)$. Now because of disjointness the inequalities may be added to obtain $n[\beta \cup \alpha, h(x)] \geqq n(\gamma \cup \alpha, x)$. This proves the lemma.

Because of the way $\sim$ was defined we obtain as a corollary not only the fact that the operation is well defined but that $B \geqq C \rightarrow A+B \geqq A+C$. As a further remark this, of course, is an extension of the usual definition for ordinary numbers.

Proposition 1. Addition is associative and commutative with $\varnothing$ as an identity

$$
\begin{gathered}
A \leqq A+B, \bar{\varepsilon}+A=\bar{\varepsilon}, \quad A+B=0 \rightarrow A=0 \text { and } B=0, \\
A+n=B+n \rightarrow A=B .
\end{gathered}
$$

Proof. Obvious.

Henceforth we let $\infty$ denote $\bar{\varepsilon}, 0$ denote $\bar{\varnothing}$ and $n$ denote the class of all $n$ element sets. It follows from an earlier remark that $A \neq \infty \rightarrow A<A+1$. Thus in one respect all $A \neq \infty$ are similar to "finite" objects (analogous to isols). Note that $B \neq 0 \rightarrow B$ $\geqq 1 \rightarrow A+B \geqq A+1$. Now assume $A$ is infinite and $A \neq \infty$. Then by Theorem 3 $(\exists H)(A<H<A+1)$. Since $H<A+1$ it follows from the previous remark that 
$\neg(\exists B)(H=A+B)$ even though $A<H$. Thus the order is not the same as the natural one induced from the semigroup operation.

Multiplication is defined as in the theory of R.E.T.'s. Let $\alpha$ and $\beta$ be representatives of $A$ and $B$ respectively. Then

$$
A \cdot B=J(\overline{\alpha, \beta}) .
$$

One difficulty in proving that multiplication is well defined is that there is no guarantee that the function $J(m, n) \rightarrow J[\alpha(m), B(n)]$ is increasing. We shall get around this by means of the "scrambling" lemma.

SCRAMBLING LEMMA. If there exists a permutation $\gamma$ such that for some recursive $h, \beta[\gamma(n)] \leqq h[\alpha(n)]$ for all $n$ then $\beta \geqq \alpha$.

Proof. Assume that $h$ is strictly increasing. Then $i \leqq n \rightarrow \beta[\gamma(i)] \leqq h[\alpha(i)]$ $\leqq h[\alpha(n)]$. Thus $\beta(i) \leqq h[\alpha(n)]$ for at least $n+1$ numbers $i$, hence $\beta(n) \leqq h[\alpha(n)]$. This completes the proof.

LEMMA. If $\beta \geqq \gamma$, then $J(\alpha, \beta) \geqq J(\alpha, \gamma)$.

Proof. Suppose that $(\exists$ recursive $h)(\forall n)[\beta(n) \leqq h \gamma(n)]$. Then

$$
(\forall m)(\forall n)\left\{J[\alpha(m), \beta(n)] \leqq h^{\prime} J[\alpha(m), \gamma(n)]\right\}
$$

where $h^{\prime}(x)=J[k(x), h l(x)]$. The argument is completed by the scrambling lemma.

A similar argument shows that if $\alpha \geqq \beta$ then $J(\alpha, \gamma) \geqq J(\beta, \gamma)$. We thus obtain not only the fact that multiplication is well defined but that $A \geqq B \rightarrow A C \geqq B C$. Note that the argument is essentially valid even if $\alpha$ or $\beta$ is finite. In that case we can use the convention that the function corresponding to a finite set is defined on an initial segment of the integers only and that the sentence $(\exists h)\{\beta(n) \leqq h[\alpha(n)]\}$ is automatically false if $\alpha$ is defined and $\beta$ is undefined and automatically true in all other cases where not both $\alpha$ and $\beta$ are defined.

Proposition 2. Multiplication is commutative, associative, and distributive with respect to addition, $A+A=2 A, A \cdot 1=A, A \cdot 0=0$.

Proof. Obvious, since the scrambling lemma takes care of the only possible difficulties.

One interesting corollary of the scrambling theorem is that $\alpha 1-1$ is reducible to $\beta \rightarrow \beta \geqq \alpha$. Hence sets of the same 1-1 equivalence type have the same density.

\section{Special subclasses of $\Delta$.}

THEOREM 4. There exists a hyperimmune set with hyperimmune complement.

Proof. Let $\alpha(0)=0$ and $\beta(0)=1$. We define $\alpha$ and $\beta$ by induction. Arrange all pairs $(f, \delta)$ in a sequence where $f$ is a recursive function and $\delta=0$ or 1 . At each pair 
$(f, \delta)$ we enlarge the domain of $\alpha$ and $\beta$ as follows. Suppose $\alpha$ is defined for $i \leqq n$, $\beta$ is defined for $i \leqq n$ and we reach the pair $(f, 0)$. Define

$$
\alpha(n+1)=\max [\alpha(n), \beta(n), f(n+1)]+1
$$

and

$$
\beta(n+i)=[\max \alpha(n), \beta(n)]+i \text { for all } i>0
$$

such that $\beta(n+i) \leqq \max [\alpha(n), \beta(n), f(n+1)]$. (Intuitively, if we think of $\alpha$ and $\beta$ as boxes, a big number is put into $\alpha$ and all previous numbers not yet used into $\beta$. Of course "poor" $\beta$ might not get anything at this step but it is sure to get numbers at other steps.) At $(f, 1)$ we reverse the role of $\alpha$ and $\beta$ in the definition.

It is clear from the construction that $\alpha$ and $\beta$ are complements and that neither $\alpha$ nor $\beta$ is recursively bounded. Q.E.D.

Corollary. $\exists A, B \in \Delta(A, B \neq \infty$ and $A+B=\infty)$.

Proof. Choose $A=\bar{\alpha}$ and $B=\bar{\beta}$ where $\alpha$ and $\beta$ are the sets constructed in the proof of Theorem 4 .

This result illustrates an important difference between densities and isols. Although as remarked earlier all $A \neq \infty$ are like finite objects, it is possible for the sum of two such elements to be $\infty$. We feel that this phenomenon of "superfiniteness" is interesting mathematically and philosophically. Stretching one's imagination one can think of such elements $A$ and $B$ as analogous to Philo's intermediaries between God and Man.

Note that since $A+\infty=\infty$ we have $A+B=A+\infty$ although $B \neq \infty$. As a reminder note that $A<A+1$.

As a result in the other direction we show

Proposition 3. $A+A=\infty \rightarrow A=\infty$.

Proof. Let $\bar{\alpha}=A$. As a representative of $A+A$ let us use the set

$$
\beta=\{2 \alpha(n), 2 \alpha(n)+1\} .
$$

Since $A+A=\infty,(\exists$ recursive $h)(\forall x)\{n[\beta, h(x)]\} \geqq x$. In particular $n[\beta, h(2 x)] \geqq 2 x$. Now by construction of $\beta$ it is clear that $2 n[\alpha, x] \geqq n[\beta, x]$. Hence $n[\alpha, h(2 x)] \geqq x$. Since the function $h(2 x)$ is recursive this completes the proof.

Corollary. $A+B=\infty \rightarrow A \cup B=\infty$.

Proof. $A \cup B \geqq A$ and $A \cup B \geqq B$. Hence $(A \cup B)+(A \cup B) \geqq A+B=\infty$. Thus by the previous result $A \cup B=\infty$. (The other direction is too trivial to even bother stating.)

We next study hyperimmune sets at the other end of the spectrum.

DEFINITION 1. $\alpha(n)$ is strongly hyperimmune if

$$
(\forall \text { recursive } h)\left(\exists n_{0}\right)\left(\forall n \geqq n_{0}\right)[\alpha(n)>h(n)] .
$$


DEFINITION 2. $\alpha(n)$ is uniformly hyperimmune if

$$
(\forall \text { recursive } h)\left(\exists n_{0}\right)\left(\forall n \geqq n_{0}\right)[\alpha(n+1)>h \alpha(n)] \text {. }
$$

All uniformly hyperimmune sets are strongly hyperimmune. Existence of uniformly hyperimmune sets is easily seen. Enumerate the recursive functions $\left\{f_{n}\right\}$ and let $g_{n}=\max _{i \leqq n} f_{i}$. Choose $\alpha(n+1)>g_{n}[\alpha(n)]$. This is clearly always possible. Then if $h=f_{i}$, we can choose $n_{0}$ to equal $i$. In future $f, g, h$ will always represent strictly increasing recursive functions.

Proposition 4. If $\beta$ is strongly hyperimmune and $\beta \geqq \alpha$, then $\alpha$ is strongly hyperimmune.

Proof. Choose $f$ so that $\beta(n) \leqq f[\alpha(n)]$. Let $h$ be arbitrary. Since $\beta$ is strongly hyperimmune $\left(\exists n_{0}\right)\left(\forall n \geqq n_{0}\right)[\beta(n)>f h(n)]$. Hence if $n \geqq n_{0}, f[\alpha(n)] \geqq \beta(n)>f h(n)$, hence $\alpha(n)>h(n)$.

COROLLARY. $\beta$ strongly hyperimmune and $\beta \sim \alpha \rightarrow \alpha$ strongly hyperimmune.

The corollary enables us to carry over the concept to densities in the obvious way, i.e. a density is strongly hyperimmune $\leftrightarrow$ its representatives are strongly hyperimmune. Let $\Delta_{s}$ be the class of all strongly hyperimmune densities. It follows from the previous proposition that $B \in \Delta_{s}$ and $A \leqq B \rightarrow A \in \Delta_{s}$.

Proposition 5. If $A \in \Delta_{s}$ and $B \in \Delta_{s}$ then $A+B \in \Delta_{s}$.

Proof. Choose $\alpha \in A$ and $\beta \in B$ such that $\alpha \cap \beta=\varnothing$. Let $\gamma=\alpha \cup \beta$. Let $h$ be arbitrary. Choose $n_{0}$ so that $n>n_{0}$ implies $\alpha(n)>h(2 n+1)$ and $\beta(n)>h(2 n+1)$. Then $\gamma(2 n) \geqq \min [\alpha(n), \beta(n)]>h(2 n+1)$. The first inequality follows from the obvious fact that the only possible elements in $\gamma$ which are less than $\min [\alpha(n), \beta(n)]$ are $\alpha(0), \ldots, \alpha(n-1), \beta(0), \ldots, \beta(n-1)$. A fortiori $\gamma(2 n+1)>h(2 n+1)$. Since $h(2 n+1)>h(2 n)$ this proves the result. Note that the above proposition together with Theorem 4 guarantees the existence of densities $\neq \infty$ in $\Delta-\Delta_{s}$. This is also easily seen directly.

We have shown that $\Delta_{s}$ is an ideal in $\Delta$. Since $A \cup B \leqq A+B$ it follows that $A \in \Delta_{s}$ and $B \in \Delta_{s}$ imply $A \cup B \in \Delta_{s}$. This can also be shown directly by means of Theorem 2.

Uniformly hyperimmune sets behave in more complicated ways. First the analogue of Proposition 4 fails. Let $\beta$ be uniformly hyperimmune. Define $\alpha$ as follows: $\alpha(2 n)=\beta(2 n)$ and $\alpha(2 n-1)=\beta(2 n)-1$. Then $\beta(n) \leqq \alpha(n)$ for all $n$ hence $B \geqq A$. However, $\alpha(2 n)=\alpha(2 n-1)+1$ so that the required condition fails already for $h(n)=n+1$. Nevertheless the corollary is still valid.

Proposition 6. If $\beta$ is uniformly hyperimmune and $\beta \sim \alpha$, then $\alpha$ is uniformly hyperimmune.

Proof. Let $h$ be arbitrary. Choose $f$ and $g$ so that

$$
\forall n\{\beta(n) \leqq f[\alpha(n)]\} \quad \text { and } \quad \forall n\{\alpha(n) \leqq g[\beta(n)]\} .
$$


Choose $n_{0}$ so that for $n \geqq n_{0}, \beta(n+1) \geqq f h g \beta(n)$. Then $f[\alpha(n+1)] \geqq \beta(n+1) \geqq f h g \beta(n)$ $\geqq f h \alpha(n)$. Hence $\alpha(n+1) \geqq h \alpha(n)$.

A density is uniformly hyperimmune $\leftrightarrow$ its representatives are hyperimmune. Let $\Delta_{u}$ be the class of all uniformly hyperimmune densities. Then $\Delta_{u} \subset \Delta_{s}$. The previous counterexample together with Proposition 4 , shows that $\Delta_{s}-\Delta_{u}$ is nonempty. Note that $A+A$ is never in $\Delta_{u}$ since a standard representative $\{2 \alpha(n)\}$ $\cup\{2 \alpha(n)+1\}$ of $A+A$ satisfies $\alpha(n+1)=\alpha(n)+1$ infinitely often. The counterexample also shows that $(\forall B)(\exists A)\left(A \leqq B\right.$ and $\left.A \notin \Delta_{u}\right)$ because $B$ may be arbitrary. Thus intuitively speaking membership in $\Delta_{u}$ is not a measure of "size". The property is analogous to exponential absorption of $X_{0}$ in the theory of cardinals, i.e. it is like a cofinality type property.

Note that $A+B \in \Delta_{u} \rightarrow A \in \Delta_{u}$. This is obvious by using representatives $\alpha$ and $\alpha \cup \beta$ of $A$ and $A+B$ respectively.

The cancellation law (Theorem 5 in the sequel) was originally proved for elements in $\Delta_{u}$. Although the importance of $\Delta_{u}$ has been diminished by the discovery that the law remains valid for any element in $\Delta_{s}$, we still feel that $\Delta_{u}$ is a significant class to study. In fact we shall prove that $\Delta_{u}$ can be characterized as the class of all infinite multiple free densities where we say that $A$ is multiple free iff $\neg(\exists$ infinite $B)(\exists C)(A=2 B+C)$.

First if $A$ has the form $2 B+C, A$ cannot be in $\Delta_{u}$ since $2 B \notin \Delta_{u}$. Conversely if $A \notin \Delta_{u}$ and $\alpha \in A$ then there exists an $h$ and a strictly increasing sequence $n_{i}$ such that $\alpha\left(n_{i}+1\right) \leqq h \alpha\left(n_{i}\right)$. We may assume that $n_{i}+1 \geqq n_{i}+2$. Then if $B=\left\{\alpha\left(n_{i}\right)\right\}, \gamma=$ $\left\{\alpha\left(n_{i}+1\right)\right\}$ and $\delta=\alpha-\beta-\gamma$, it follows that $\bar{\beta}=\bar{\gamma}$ and hence that $A=2 \bar{\beta}+\bar{\delta}$.

We now prove the important cancellation law for densities in $\Delta_{s}$.

Theorem 5. $A \in \Delta_{s}$ and $A+B \geqq A+C \rightarrow B \geqq C$, hence $A \in \Delta_{s}$ and $A+B=A+C$ $\rightarrow B=C$.

Proof. Choose $\alpha \in A, \beta \in B$, and $\gamma \in C$ so that $\alpha \cap B=\alpha \cap \gamma=\varnothing$ and choose $h$ so that $n[\alpha \cup B, h(x)] \geqq n[\alpha \cup \gamma, x]$ for all $x$. Our only hurdle is the possible existence of elements in $\alpha$ greater than $x$ but not greater than $h(x)$. We shall get around this by using the function $h^{i}(x)$, the $i$ th iterate of $h(x)$. Of course $h^{x+1}(x)$ is strictly increasing recursive.

Since $A \in \Delta_{s},\left(\exists x_{0}\right)\left(\forall x \geqq x_{0}\right)\left[\alpha(x)>h^{x+1}(x)\right]$, i.e. there are at most $x$ elements in $\alpha$ which are $\leqq h^{x+1}(x)$. Hence among the $x+1$ intervals, $x<y \leqq h(x), h(x)<y$ $\leqq h^{2}(x) \cdots h^{i}(x)<y \leqq h^{i+1}(x) \cdots h^{x}(x)<y \leqq h^{x+1}(x)$ at least one has no elements in $\alpha$. Suppose no elements $y \in \alpha$ satisfy $h^{i}(x)<y \leqq h^{i+1}(x)$. Now $n\left[\alpha \cup \beta, h^{i+1}(x)\right]$ $\geqq n\left[\alpha \cup \gamma, h^{i}(x)\right]$. We can conclude that $n\left[\beta, h^{i+1}(x)\right] \geqq n\left[\gamma, h^{i}(x)\right]$ hence a fortiori $n\left[\beta, h^{x+1}(x)\right] \geqq n[\gamma, x]$. We know this only for $x \geqq x_{0}$ but fortunately this suffices. Hence $\beta \geqq \gamma$ and thus $B \geqq C$.

\section{BIBLIOGRAPHY}

1. Yu. T. Medvedev, On nonisomorphic recursively enumerable sets, Dokl. Akad. Nauk SSSR 102 (1955), 211-214. 
2. E. A. Pavlova, Denseness of hyperimmune sets, Dokl. Akad. Nauk SSSR 139 (1961), 814-817 = Soviet Math. Dokl. 2 (1961), 1017-1020.

3. - The lattice of denseness of sets of natural numbers, Izv. Moldavsk. Fil. Akad. Nauk SSSR 1960, no. 10 (76), 31-38.

4. H. G. Rice, On the relative density of sets of integers, Proc. Amer. Math. Soc. 8 (1957), 320-321.

Rutgers, The State University,

New Brunswick, NeW Jersey

COMPUTER SCIENCES,

El Segundo, California 